\title{
Shape Memory and Pseudoelastic Properties of Fe-Mn-Si and Ti-Ni Based Alloys
}

\author{
G. Guénin
}

Institut National des Sciences Appliquées de Lyon, G.E.M.P.P.M., bâtiment 502, 69621 Villeurbanne, France

\begin{abstract}
The aim of this presentation is to analyse and discuss some recent advances in shape memory and pseudoelastic properties of different alloys. Experimental work in connection with theoretical ones will be reviewed. The first part is devoted to the microstructural origin of shape memory properties of Fe-Mn-Si based alloys ( $\gamma-\varepsilon$ transformation); the second part is a synthetic analysis of the effects of thermomechanical treatments on shape memory and pseudoelastic effects in $\mathrm{Ti}-\mathrm{Ni}$ alloys, with some focus on the behaviour of the $R$ phase introduced.
\end{abstract}

\section{SHAPE MEMORY OF $\gamma-\varepsilon$ IRON BASED ALLOYS}

Until eightie's it was generally thought that full shape memory effect was the privilege of alloys undergoing thermoelastic martensitic transformation [1]. Only very incomplete shape memory has been found at this time in austenitic iron based alloys which exhibit non thermoelastic $\gamma-\alpha^{\prime}$ (f.c.c. - b.c.c. or b.c.q.) or $\gamma-\varepsilon$ (f.c.c. - h.c.p.) martensitic transformation. The FezPt ordered alloy was an exception but in this case, the $\gamma-\alpha^{\prime}$ transformation becomes thermoelastic. A good shape memory implies that no permanent slip is produced during the transformation and that the transformation on heating occurs reversibly like a movie projected in reverse way. In case of $\gamma-\alpha^{\prime}$ transformation, it seems that if no slip occurs, a good shape memory is obtained, that is to say that the second condition is fulfilled. The $\gamma-\alpha^{\prime}$ martensitic transformation should be inherently reversible. This is demonstrated by the case of Fe-Ni-C [2] and Fe-Ni-Ti-Co [3] which can exhibit nearly perfect shape memory when the slip is inhibited by some hardening due respectively to ausforming and ausageing.

The case of $\gamma-\varepsilon$ martensitic transformation, on which it will be focussed here, is somewhat different because the crystallographic reversibility does not imply the shape reversibility. Indeed the $\gamma-\varepsilon$ occurs by the introduction of a stacking fault every second plane on the f.c.c. close packed planes. Three fault vectors in each plane (at $120^{\circ}$ of each other) are able to produce the same $\varepsilon$ crystal orientation with the same $\varepsilon-\gamma$ interface (habit plane : the close packed plane itself), once the $\varepsilon$ plate formed, There is no way to know what fault vector (or combination of fault vectors) has formed the $\varepsilon$ plate. Yet each fault vector lead to a different shape change produced by the $\varepsilon$ martensite plate. The reverse transformation (reverse faulting) can occur by other fault vector(s) leading to the same $\gamma$ crystal orientation but not the same shape as initially. The crystal orientation is recovered but not the shape. This can be experimentally shown by the observation of surface relief [4]. As a consequence, for good shape memory properties of $\gamma-\varepsilon$ alloys the two conditions, no slip and reversibility of the transformation, have to be improved.

Sato et al [5] have found a nearly complete shape memory effect in a single crystal of Fe-30Mn$1 \mathrm{Si}$ when the deformation was performed in such a way to activate only one fault vector. This kind of alloy is far from the thermoelasticity, in particular the hysteresis of the transformation is about $150 \mathrm{~K}$. Murakami et al [6] has improved the compositions of this alloy to obtain a complete shape memory effect for polycrystals. Their conclusion was that the addition of silicon has three benefits : it reduces the Neel temperature of austenite (para-antiferromagnetic transformation) which must be below the $\mathbf{M}_{\mathbf{s}}$ temperature for no interference with the martensitic transformation; it raises the strength of the mattrix; it lowers the fault energy of the austenite (favours the $\gamma-\varepsilon$ transformation in relation to the $\gamma-\alpha^{\prime}$ transformation). This kind of alloy and its derivatives to improve the corrosion resistance (Fe-Mn-Si- 
$\mathrm{Cr}-\mathrm{Ni}$ ) have been widely studied these last years. The main feature is that the martensitic transformation characteristics as well as the shape memory properties are, for a given alloy, extremly sensitive to the thermomechanical treatment and to the microstructure. A successful treatment to improve the shape memory effect is the so called "training" [7] which consist on the repetition of $2.5 \%$ deformation at room temperature just above $\mathbf{M}_{\mathrm{s}}$, and recovery at $873 \mathrm{~K}$. The shape memory effect is improved as shown on figure 1. This is associated to the fact that this treatment increases the critical stress for slip deformation and lowers the critical stress for martensite formation (figure 2).

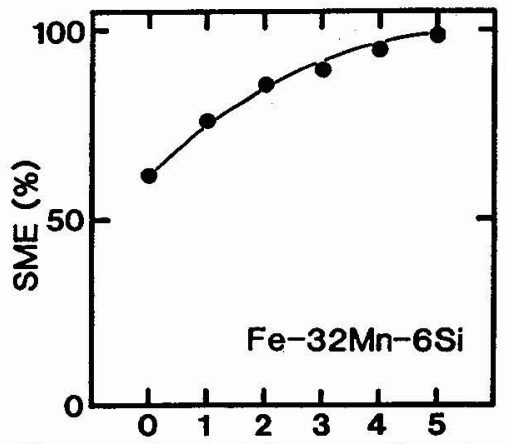

The number of times of "training"

Figure 1 : Effect on "training" on Shape Memory Effect (after [7])

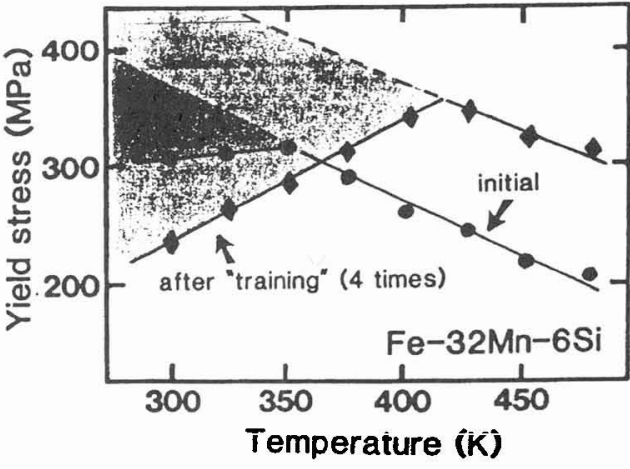

Figure 2: Effect of "training" on temperature dependance of yield stress. Positive slope corresponds to martensite formation, negative to slip deformation (after [7])

Other less complicated kind of treatment has been proposed by Federzoni [12] in the case of Fe-Mn-Si$\mathrm{Cr}$-Ni alloy. The alloy first annealed at $823 \mathrm{~K}$ is strained about $7 \%$ at room temperature just above $\mathbf{M}_{\mathrm{s}}$, this induces some $\varepsilon$ martensite and slip deformation. It is then anealed at $973 \mathrm{~K}$ for a complete reversion of martensite and restauration of dislocations (no recrystallization). This treatment leads to a maximum fraction of martensite by cooling to liquid nitrogen temperature and leads to a good improvement of the shape memory effect (figure 3). The common features of these treatments is the presence in the parent phase of numerous dislocations and stacking faults which increase the strength of the matrix and favours the $\varepsilon$ martensite nucleation.

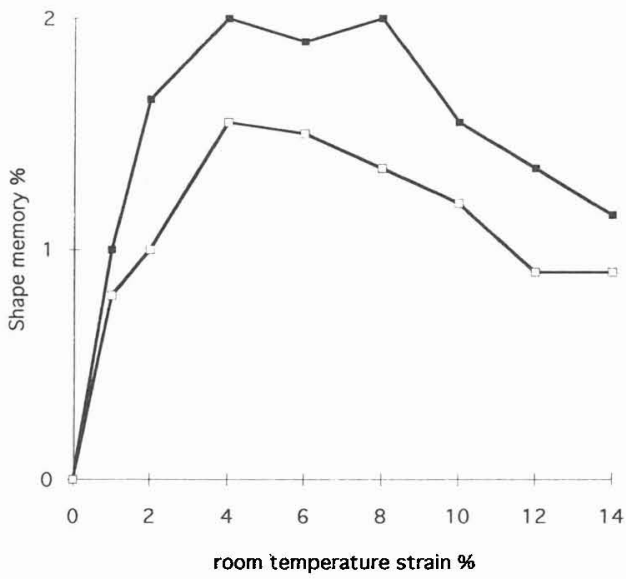

Figure 3 : Improvement of SME by treatment proposed in [12] (full squares) compared to well annealed (open squares)

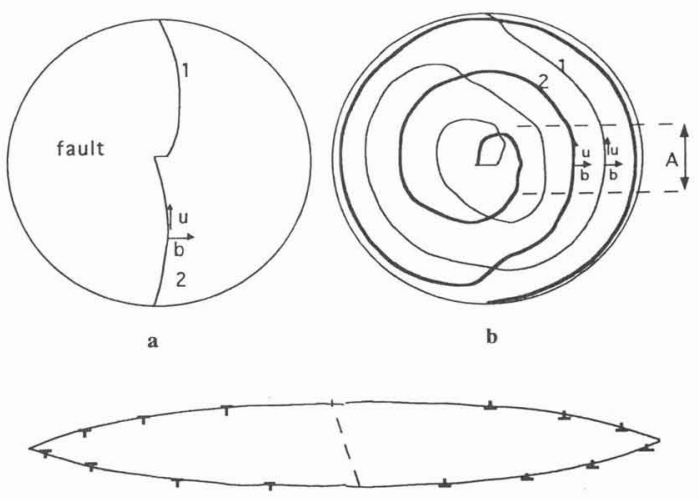

Figure 4 : Geometry of a Seeger like source limited by a grain boundary, $a$ : initial state, $b$ : grown up plate, below: the plate seen from the side (after [17]) 
The question which arises is : what transformation mechanism is able to explain the full reversibility of the stress induced martensite and how this mechanism is favoured by the thermomechanical treatment and the corresponding microstructure. Ogawa et al [8] have studied the structure of stress induced martensite in both thermomechanically treated and no treated samples. They have shown that, in the case of treated samples the martensite appears at nanometric scale as a mixture of thin martensite plates and very thin austenite lamellas. This structure is not seen in no treated sample where martensite plates include number of stacking faults. Moreover Kikuchi et al [9] have clarified the growth process : some extremely thin plates appear randomly distributed through the grain and the transformation proceeds by successive formation of other thin plates close to the first ones. These features have been confirmed by Bergeon et al $[10,11]$ in addition to the fact that the plates are clearly shown to be formed by single fault vector ( by propagation of one kind of partial dislocation) : the thin plates are monopartial.

Number of mechanisms have been proposed to explain the martensitic transformation $[13,14,15]$ but only the pole mechanism first proposed by Seeger [15] is able to explain the observayions. Besides a direct observation has been made by Hoshino et al in a single crystal of Fe-MnSi previously thermomechanically treated [16]. The good reversibility implies that this mechanism goes back way by heating. Guenin [17] has described a model to explain this reversibility (figure 4). The interaction of the same partial dislocations with grain boundaries is quasi identical to a pile up and leads to a back stress which limitates the plate thickness and which by heating initiate the way back of the partial dislocations and therefore the perfect reversibility. The interaction of thin stress induced martensite plates with grain boundaries has been observed by Bergeon et al [11] in case of $\mathrm{Fe}-\mathrm{Mn}-\mathrm{Si}-\mathrm{Cr}-$ $\mathrm{Ni}$ alloy themomechanically treated. It is found that for martensite induced by low strains $(1 \%)$, that is to say for good reversibility and shape memory effect, thin plates ends on grain boundaries becoming thiner and thiner (figure 5), the angle of the tip is very small corresponding to spaced partial dislocations.
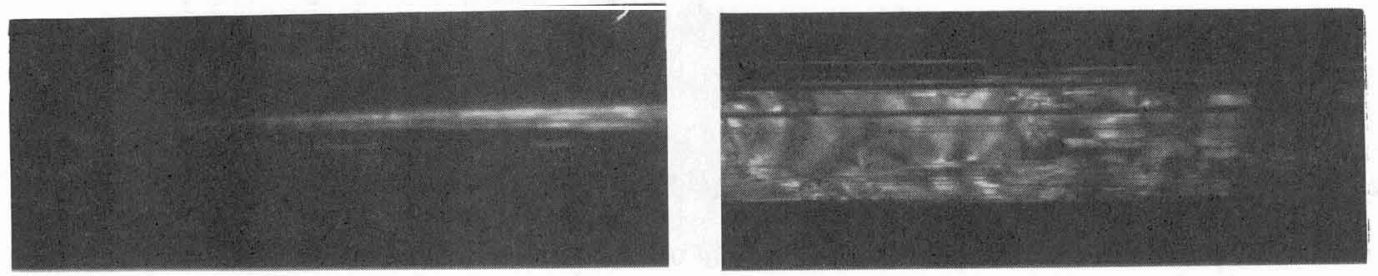

Figure 5 : change of thickness of martensite plate inside the grain : on the right middle of the grain, on the left close to the grain boundary visible at the tip (dark field on martensite, $2 \mathrm{~cm}=250 \mathrm{~mm}$, after [11])

Larger strains lead to several relaxation phenomena : initiation of thin $\varepsilon$ plates on second $\{111\}$ plane; formation of triangular mixture of $\gamma$ phase and secondary $\varepsilon$ thin plates (figure 6). These relaxation phenomena suppress the back stress for the initial partial dislocations. Therefore the reversion of the martensite plates by heating can occur by other nucleation mechanism instead of initial dislocations return, leading to a poor shape memory. This analysis is coherent with the macroscopic observations of the reversion of the $\varepsilon$ martensite during the heating. The reversion of stress induced martensite spreads over a large temperature domain, if compared with martensite induced by cooling, in two steps for low strains and one step at higher temperature for larger strains. The reversion process is therefore delayed by the relaxation on grain boundaries (or other obstacles). Moreover, during the heating a given fraction of reverted $\varepsilon$ martensite leads to a larger shape recovery at low temperature than at high temperature [12]. This implies the the reversibility is better at low temperature (thin plates with no relaxation) than at high temperature (larger plates with stress relaxation).

As a conclusion, a good shape memory will be attained if no relaxation, essentially at grain boundaries, is induced by the strain. This implies that very thin $\varepsilon$ plates must only be produced. A good thrmomechanical treatment is the one which leads to numerous nucleation sites that is to say favorable situations for pole mechanism : partial dislocations and pole dislocations. These dislocations are also able to avoid the normal slip.

These kind of alloys are well adapted for bounding applications as cheaper substitute to $\mathrm{Ni}-\mathrm{Ti}$ ones. For example junction of tubes have been developped by Nippon Steel and a project by the same company is running for junction of elements in tunnels where small space is avaitable. 


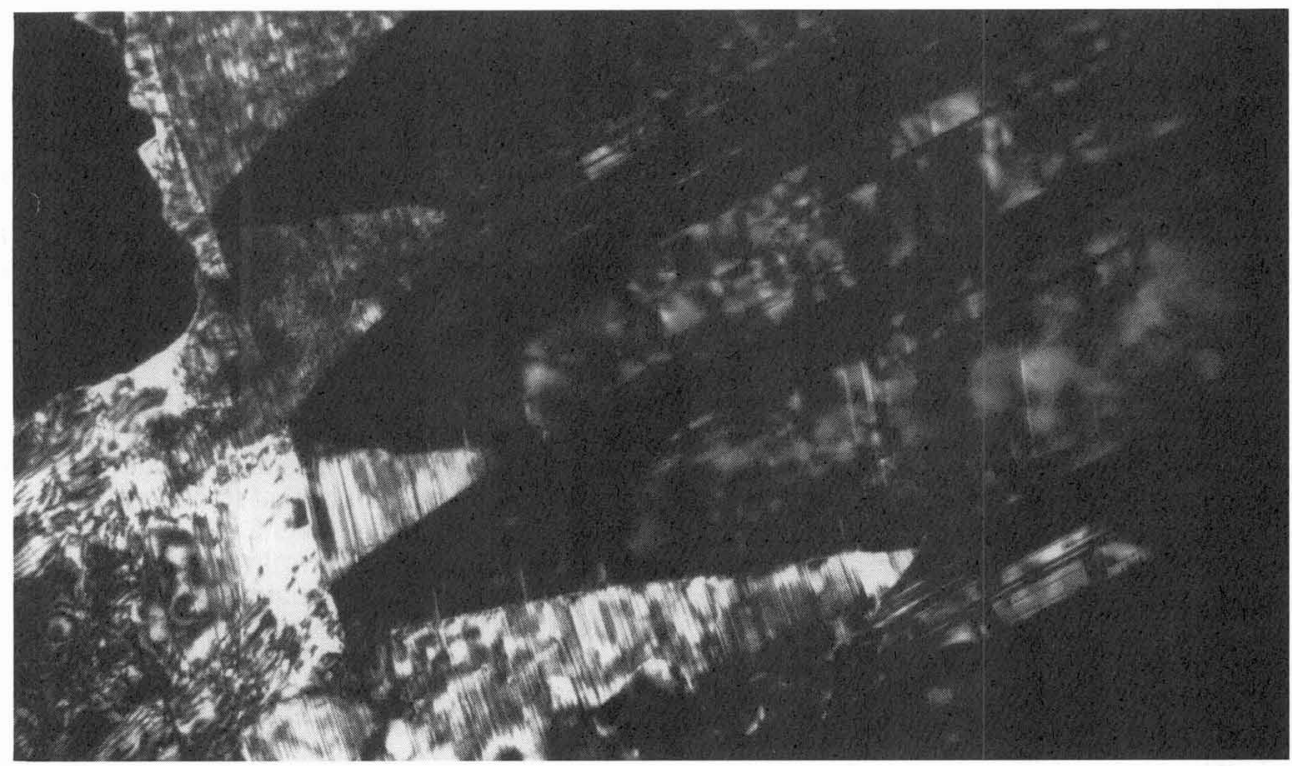

Figure 6 : typical relaxation phenomena at grain boundary for large strains : triangular mixture of $\gamma$ phase and secondary $\varepsilon$ thin plates to accomodate the strains at the tip of the principal martensite plates, in dark. $(2 \mathrm{~cm}=250 \mathrm{~nm}$, after $[11])$

\section{SHAPE MEMORY AND PSEUDOELASTIC PROPERTIES OF Ti-Ni ALLOYS}

The pseudoelastic (or superelastic) behaviour of well solution treated binary $\mathrm{Ti}-\mathrm{Ni}$ alloy is hardly observed, this is due to the combined effect of rather large hysteresis of the transformation and rather low yield stress for conventional slip deformation [19]. To a lower extent the shape memory quality is also dependent on the yield stress. A complete pseudoelastic behaviour is only seen for high Ni content in a narrow temperature domain [20]. The way to improve this behaviour is to rise the yield stress of the matrix by some different treatments like precipitation hardening or cold working and moderate annealing. These ways have been explored in early eightie's. Precipitation hardening can be achieved, when the $\mathrm{Ni}$ content is high, by ageing at moderate temperatures (573-773K) after solution treatment. This leads some coherent $\mathrm{Ti}_{3} \mathrm{Ni}_{4}$ precipitates to appear. Figure 7 [21] shows the variations of critical stresses for inducing martensite $\sigma_{M}$ and for complete reverse transformation $\sigma_{R}$ corresponding to several Ti-Ni alloys submitted to solution treatment followed by ageing at $673 \mathrm{~K}$. It can be seen that for high $\mathrm{Ni}$ content ( 51.6 and $\mathbf{5 0 . 6}$ at\%) a perfect pseudoelastic behavour occurs (linear parts of $\sigma_{\mathrm{M}}$ and $\sigma_{\mathrm{R}}$ ) in a rather wide temperature range ( $50 \mathrm{~K}$ for the $51.6 \mathrm{at} \% \mathrm{Ni}$ alloy). The quasi equiatomic alloys (49.8 and 50.1 at\% Ni) are not significantly changed by the ageing and do not exhibit any region with reverse transformation (no $\sigma_{\mathrm{R}}$ ). However if these last alloys are cold worked and then annealed at the same moderate temperature a perfect pseudoelasticity is induced as shown on figure 8 [21]. Therefore the precipitation hardening and the cold work + annealing are able to improve the pseudoelastic properties (as well as shape memory) of Ti-Ni alloys. However this has been done with a large increase of complexity : the temperatures and other characteristics of the martensitic transformation are changed in relation to the ones of the well solution treated alloys; the intermediate so-called $R$ phase transformation appears with its proper transformation temperatures, small hysteresis and thermomechanical behaviour. The figure 9 [22] shows an example of the influence of cold work + anealing on the transformation temperatures measured by electrical resistance : The $\mathbf{M}_{\mathbf{S}}$ and $\mathbf{M}_{\mathbf{f}}$ are lowered, the hysteresis of the martensitic transformation is larger, the $R$ phase begins to appear at $T_{R}$ on cooling and disappears with a small hysteresis (few degrees). The increase of resistance on cooling prior to $\mathbf{M}_{\mathbf{S}}$ in solution treated alloy and between $T_{R}$ and $T_{R}$ in treated sample is attributed to an incommensurate phase [23] without consequences on shape memory or pseudoelastic properties (no homogeneous lattice deformation). The shape memory and pseudoelastic properties of such thermomechanically treated $\mathrm{Ti}-\mathrm{Ni}$ alloys are highly 
dependent on the two involved transformations and their relative interactions. The precipitates and the lattice defects involved can themselves have a particular influence.

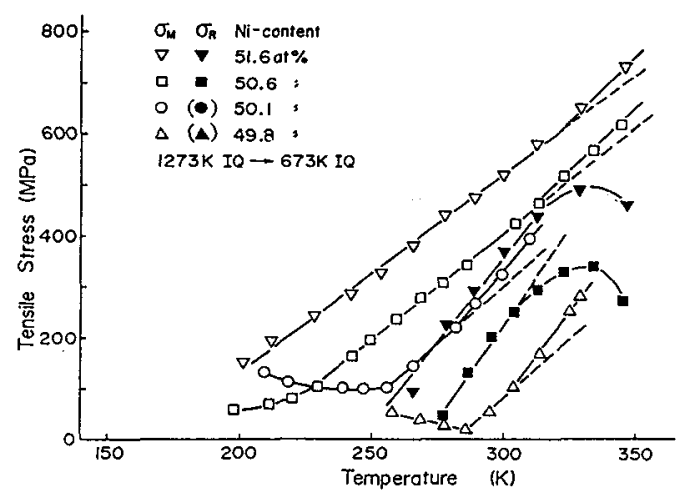

Figure 7 : Effect of solution treatment + annealing at $673 \mathrm{~K}$ on critical stresses for inducing martensite $\sigma_{M}$ and reverse transformation $\sigma_{R}$ (after $[21]$ )

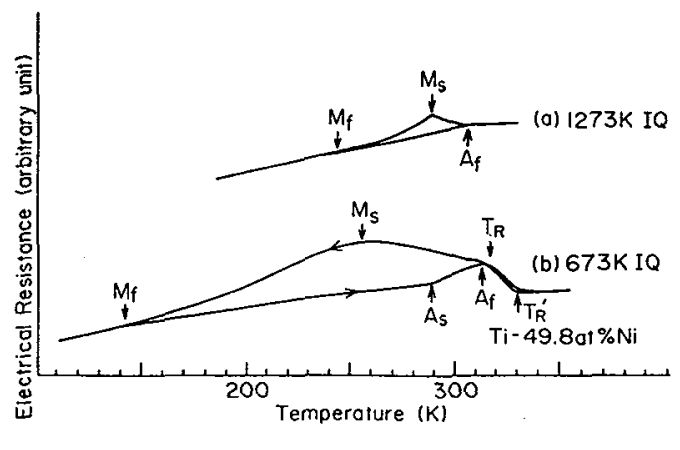

Figure 9 : Electrical resistance of $\mathrm{Ti}-49.8$ at $\% \mathrm{Ni}$ a : solution treated

b : cold worked + annealed at $673 \mathrm{~K}$ (after [22]
(B)

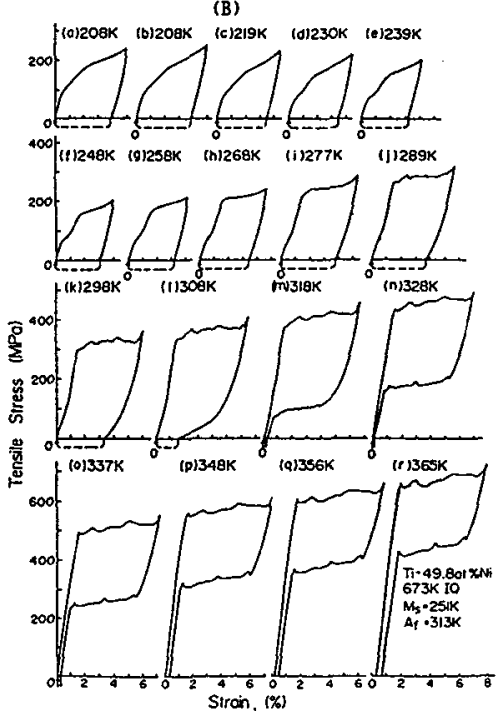

Figure 8 : Effect of previous treatment on stress-strain curves as a function of temperature in a Ti-49.8 at.\% Ni alloy. A : solution treated + annealed at $673 \mathrm{~K} ; \mathrm{B}:$ cold worked + annealed at $673 \mathrm{~K}$ (after [21]

Before to understand well the memory and pseudoelastic properties, it is important to have a knowledge of the relationships between the $\beta$ phase, the $R$ phase and the martensite : transformation sequences on cooling and heating. It is possible to well separate the two transformations by adding a third element like $\mathrm{Fe}$ in substitution to $\mathrm{Ni} \mathrm{[23].} \mathrm{In} \mathrm{this} \mathrm{case,} \mathrm{the} \mathrm{transformation} \mathrm{sequences} \mathrm{are} \mathrm{clearly}$ $\beta \rightarrow R \rightarrow M$ on cooling and $M \rightarrow R \rightarrow \beta$ on heating. However, due to the large differences between the hysteresis of the martensite and the $R$ phase transformations some complicated situations can occur as shown on figure 10 [24]. When a complete cycle is performed, the transformation sequence on cooling is $\beta \rightarrow R \rightarrow M$ and $M \rightarrow \beta$ on heating. When the cycle is limited on cooling to $\beta \rightarrow R$ transformation, the 
$R \rightarrow \beta$ is observed (curve 1 ) and occurs several degrees above the direct transformation which is below the $M \rightarrow \beta$ reverse transformation due to the large hysteresis of the martensitic transformation. When $\boldsymbol{\beta} \rightarrow \mathrm{R} \rightarrow$ partial $M$ is achieved the reverse transformation occurs in two steps : $R \rightarrow \boldsymbol{\beta}$ from untransformed $R$ on cooling and $M \rightarrow \beta$ at higher temperature.

To understand the shape memory and pseudoelastic properties in such complicated situations, it is first necessary to precise these properties for the $\mathrm{R}$ phase transformation alone, because of the quasi martensitic nature of this transformation [25]. The $\beta \rightarrow R$ transformation corresponds to a rhombohedral elongation of the cubic $\beta$ cell along $<111>$ direction and there is a gradual change of the characteristic angle $\alpha$ of the rhombohedra with decreasing temperature from $T_{R}$ [25] showing a second order transformation feature; a very small thermal hysteresis occurs ( 1 to $5 \mathrm{~K}$ depending on cases). Four variants twin related ( $\{110\}$ and $\{100\}$ twinning planes) are then observed which can form self accomodated groups [26]. The shape memory in single crystal has been described by Miyasaki et al [26, 27]. On cooling, multivariant is induced which can be transformed in single variant by the application of the stress which lead to a strain governed by the orientation of the stress and the $\alpha$ angle at the given temperature below $T_{R}$. The remove of the stress does not suppress the single variant of $R$ phase and on heating the single crystal remains until the $\alpha$ angle becomes 90 , leading to the $\beta$ phase. The shape memory mechanism is therefore somewhat different from the one of classical martensite : it corresponds to the change of $\alpha$ angle and does not involve any interface movements. The pseudoelastic and memory behaviour has been characterised for Polycrystal [22] as well as for single crystal [27] of Ti-50.5at\% Ni (precipitation hardened). The figure 11 in the case of single crystal shows the stress-strain behaviour as a function of temperature. It is clear that the strain induced by the reorientation of $R$ phase $\left(T<T_{R}\right)$ decreases with increasing temperature according to the $\alpha$ changes, a nice close correlation has been established. Just above $T_{R}$ a pseudoelastic behaviour is observed but with a very small strain : about $0.05 \%$ at $T_{R}+10 \mathrm{~K}$. This must be compared to about $1 \%$ for reorientation at $T_{R}-60 \mathrm{~K}$. It also corresponds to a very steep critical stress versus temperature curve. The behavour of the polycrystal is very similar but with smaller strains (the single crystal of figure 11 is favourably oriented).

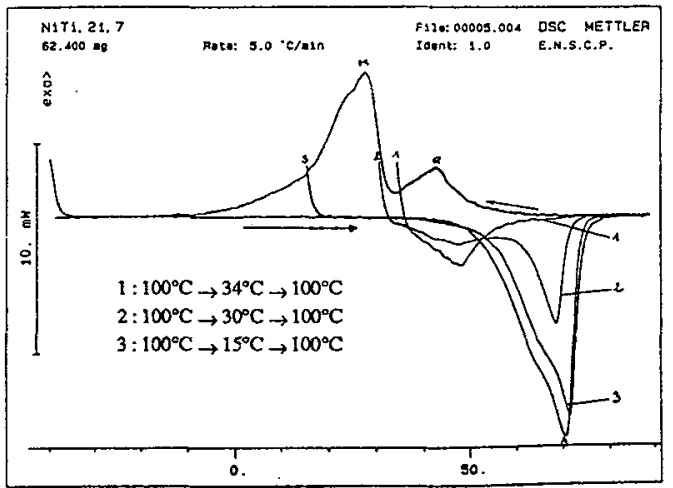

Figure 10 : DSC thermograms obtained by using complete and partial thermal cycling (after [24]
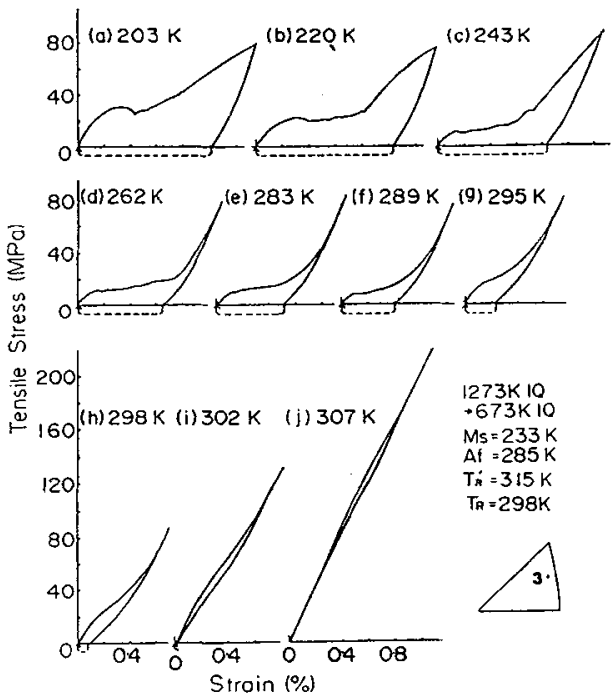

Figure 11 : Stress-strain curves for single crystal of Ti-50.5 at.\% $\mathrm{Ni}$, precipitation treated. Dashed lines show the strain recovery upon heating after unloading (after [27]) 
The critical stress versus temperature has been analysed in terms of Clausius-Clapeyron equation for the case of Ti-Ni-Fe alloy [28], it is found that the transformation enthalpy evaluated by this way is much smaller than the one directly measured by calorimetry [29] : $54.7 \mathrm{~J} . \mathrm{mol}^{-1}$ versus $431 \mathrm{~J}^{-\mathrm{mol}^{-1}}$ even if this last value seems to be overestimated if compared with more accurate result $\left(285 \mathrm{~J}^{\mathrm{mol}}{ }^{-1}\right.$ from Manosa et al[30]). The authors [28] postulates that this discreapancy could be due to the fact that the enthalpy measured by Clausius-Clapeyron analysis is only due to the $\mathbf{R}$ phase transformation whereas the enthalpy measured by calorimetry involves the incommensurate and $R$ phase transformations . This is supported by recent investigation [30] showing double peak thermograms, a higher temperature peak with no hysteresis attributed to $\beta \rightarrow$ incommensurate phase and a lower temperature peak with $1.8 \mathrm{~K}$ hysteresis attributed to incommensurate phase $\rightarrow R$; however the enthalpies seem to be of the same order of magnitude and this is not sufficient to explain the discreapancy which remains an open problem. From these studies, it seems that the pseudoelastic behaviour due to the $R$ phase is very small $(0.05 \%$ to $0.2 \%)$ and occurs in a small temperature range (10K) because of the very steep critical stress versus temperature curve.

For applications the stress assisted two way memory effect is interesting and the $\mathbf{R}$ phase is able to give such a behaviour as demonstrated by Tamura et al [31] and Todoroki [32] : figure 12. It is seen that in case of cold worked + annealed Ti-Ni a reversible strain of about $0,5 \%$ is obtained with a remarquable small hysteresis under a quasi constant stress of about $100 \mathrm{MPa}$. The stability under cycling is excellent : no noticeable change with $510^{5}$ cycles.

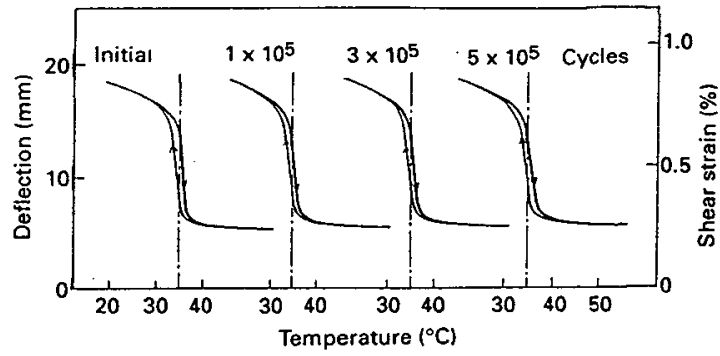

Figure 12 : Stress assisted two way memory effect due to $R$ phase in a spring during thermal cycling between $20^{\circ} \mathrm{C}$ and $55^{\circ} \mathrm{C}$ with a stress of about $100 \mathrm{MPa}$ (after [32])

Pseudoelastic and shape memory behaviour for the combined $R$ phase and martensitic transformations :

Many situations can be encountered even for a given alloy with a precise given thermomechanical treatment. These situations can be analysed with the help of stress versus transformation temperatures diagram figure 13 [32].

Stress-strain behaviour at different temperatures has been analysed by Miyasaki and Otsuka $[22,28]$ figure 14 . At least six different behaviours are possible : range a $\left(T<M_{f}\right)$ sample fully martensitic only one stage corresponding to martensite reorientation, the remaining strain is recovered by heating; range $b\left(M_{\mathrm{f}}<\mathrm{T}<\mathrm{M}_{\mathrm{S}}\right)$ sample with martensite and $\mathrm{R}$ phase, two stages are present corresponding to reorientation of $\mathbf{R}$ phase and induction and reorientation of martensite; range $c$ $\left(M_{S}<T<A_{f}\right)$ the sample is fully $R$ phase, two stages are present corresponding to reorientation of $R$ phase and to induction of oriented martensite by stress from the $R$ phase; range $d$ ( $A_{f}<T<R_{f}$ ) the sample is fully $\mathbf{R}$ phase which is first reoriented then pseudoelastic behaviour occurs corresponding to transformation : oriented $R$ phase $\rightarrow$ oriented martensite $\rightarrow$ oriented $R$ phase; range $e\left(A^{*}<T<T_{x}\right)$ the sample just above $A *$ is $\beta$ phase, pseudo elasticity from $R$ phase alone can be seen (small amplitude) as well as pseudoelasticity from martensite; range $f\left(T>T_{x}\right)$ only the pseudoelasticity from martensite can be seen.

The strain-temperature behaviour with constant stress has been described by Todoroki [32]. It depends on the stress level and the temperatures involved. Some situations are shown on the figure 15 and can be understood from figure $13 ; 1 \rightarrow 2 \rightarrow 1$ cycle involves only $\beta$-martensite transformation; $3 \rightarrow 4$ 
$\rightarrow 3$ or $7 \rightarrow 8 \rightarrow 7$ cycles involves only $\beta-R$ phase transformation; $3 \rightarrow 6 \rightarrow 3$ cycle involves $\beta-R$ phasemartensite transformations on cooling and martensite $\beta$ on heating.

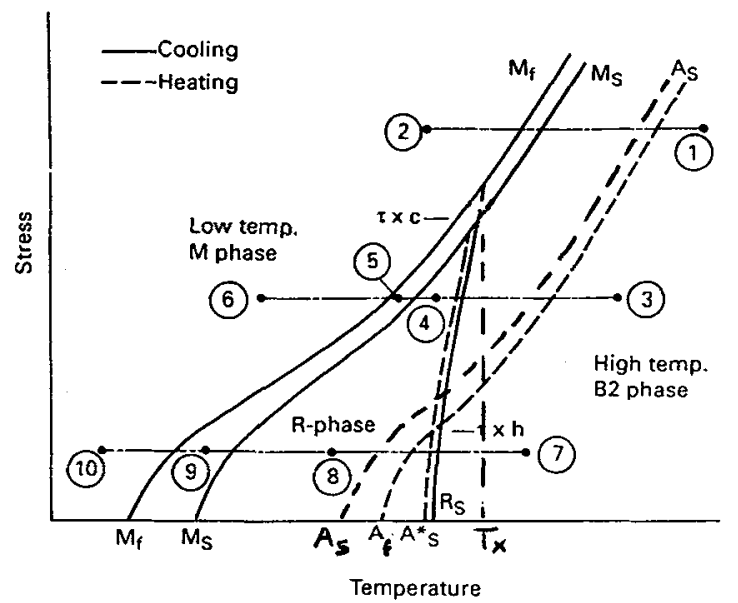

Figure 13 : Typical stress-temperature phase diagram. $R_{s}$ line is the transformation start for $R$ phase on cooling, $A *$ line is the transformation start temperature for $R$ phase on heating, the $R_{f}$ and $A *_{f}$ lines have not been represented for sake of simplicity (after [32])

(a) $T<M_{f}$
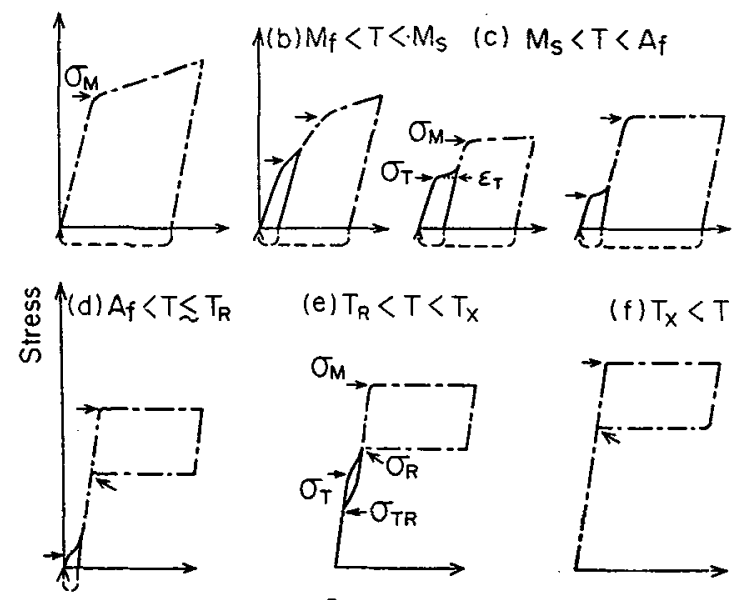

(e) $T_{R}<T<T_{X}$

$(f) T_{x}<T$
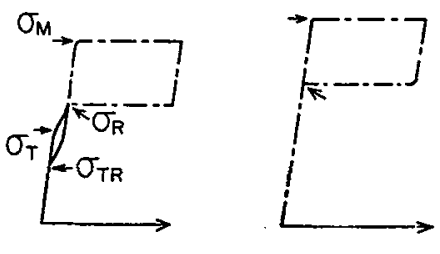

Strain

Figure 14 : Classification of stress-strain curves according to test temperatures for Ti-Ni alloys (after [22])

As can be seen the stress-strain as well as strain-temperature curves are really delicate to analyse in details. The stress versus transformation temperatures diagram (figure 13) is the essential tool to delimitate the different regimes, but it is far to be sufficient to predict the curves because the strain informations are not included. For example, as shown before, the maximum recoverable strain involved for the $\mathrm{R}$ phase depends on temperature (change of lattice cell parameters); the maximum recoverable 
strain involved for the martensitic transformation itself also depends on temperature in opposite way as shown in ref.[22].

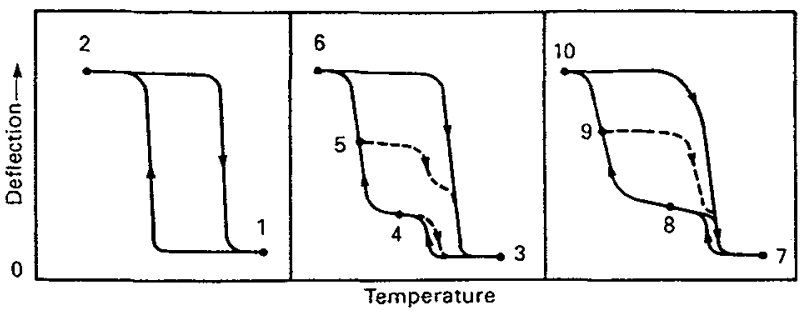

Figure 15 : Two way strain-temperature curves with constant stress corresponding to three traces on Figure 13 (after [32])

If now for a given $\mathrm{Ti}-\mathrm{Ni}$ alloy different thermomechanical treatments are applied a quasi-infinite number of situations can be encountered depending on the absolute and relative temperature positions of the martensite and $\mathrm{R}$ phase transformations. It must also be emphasized that these treatments have some influence on the hysteresis and the minimum stress to induce the reorientation (especially for martensite) see for example [22]. These last years systematic numerous precipitation and cold work + anneal experiments have been performed to find the best conditions for such application or other. For example Treppmann et al [33] have studied two Ti-Ni alloys, one of them close to the equiatomic composition and the other Ni rich (50.8 at\%). The aim is to distinguish the effects of exclusive precipitation with Nirich alloy of exclusive restauration or recristallisation (equiatomic) and the combined effects of precipitation and recristallisation (Ni rich). Temperature-time-precipitation or recristallisation have been drawn, in addition with tables giving the positions of the different transformation peak temperatures. The maximum changes have been obtained with combined reactions in Ni rich alloy.

These last years a big effort has been done to obtain Ti-Ni alloys as thin foils to enter in miniature devices or so called smart materials $[34,35]$. The knowledge of the different possible thermomechanical behaviours is essential for these devices as well as the boundary conditions for such behaviour or the other. In this case it seems that the more convenient ways to explore the stress versus transformation temperatures diagram (figure 13) are the strain-temperatures curves with constant stress. It also often corresponds to the use for the applications.

\section{GENERAL CONCLUSION}

In Ti-Ni alloys, the shape memory and pseudoelastic behaviours are inherent to the martensitic transformation, however thermomechanical treatments are necessary to improve theses properties. This implies a much larger degree of complexity in particular with the introduction of quasi martensitic $\mathbf{R}$ phase, but also gives a very wide field of possible shape memory and pseudoelastic adjustments for specific applications.

In iron based alloys the $\gamma-\varepsilon$ transformation is not able to give intrinsically a good shape memory. The thermomechanical treatments are always needed in such a way that the reverse transformation occurs along the same path to produce the shape memory.

\section{References}

[1] Wayman C.M., New aspects of martensitic transformation (Sup. to Trans. JIM, vol. 17, 1976) pp.

$159-1702$

[2] Kajiwara S., Trans. JIM, 26 (1985) 595-96

[3] Maki T. and Tamura I., Proc. ICOMAT 86, The Japan Institute of Metals (1987) pp.963-70

[4] Maki T. and Tsusaki K., Proc. ICOMAYT 92, Monterey Institute for Advanced Studies (1993)

pp.1151-62

[5] Sato A., Chishima E., Soma K. and Mori T., Acta Met., 30 (1982) 1177-83

[6] Murakami M., Otsuka H., Suzuki H.G. and Matsuda S., Proc. ICOMAT 86, The Japan Institute of Metals (1987) pp.985-90

[7] Otsuka H., Murakami M. and Matsuda S., MRS Int'l. Mtg. on Adv. Mats., 9 (1989) 451-56

[8] Ogawa K. and Kajiwara S., Materials transactions JIM, 34 (1993) 1169-76

[9] Kikuchi T., Kajiwara S. and Tomota Y., J. de Phys., C8,5 (1995) pp.445-50 
[10] Bergeon N., Thesis, oct.96, INSA Lyon, France

[11] Bergeon N., Esnouf C. and Guenin G., to be published

[12] Federzoni L., Thesis, INSA Lyon 1993 and Proc. ICOMAYT 92, Monterey Institute for Advanced Studies (1993)1175-80

[13] Bollman W., Acta Metall., 9 (1961) 972-75

[14] Fujita H., Ueda S., Acta Metall., 20 (1972) 759-67

[15] Seeger A., Z. Metallkunde, 44 (1953) 247-55

[16] Hoshino Y., Nakamura S., Ishikawa N., Yamaji Y., Matsumoto S., Tanka Y. and Sato A., Mat. Trans. JIM, 33 (1992) 253-62

[17] Guénin G.,J. de Phys., C8, 5 (1995) 433-38

[18] Delamotte E. and Altstetter C., Trans. AIME, 245 (1969) 651-59

[19] Otsuka K. and Shimizu K., Internat. Met. Rev., 31 (1986) 93-114

[20] Saburi T., Tatsumi T. and Nenno S., J. de Phys, C4,43 (1982) 261-266

[21] Miyasaki S., Ohmi Y., Otsuka K. and Suzuki Y., J. de Phys, C4,43 (1982) 255-260

[22] Miyasaki S. and Otsuka K., Met. Trans. A, 17 (1986)53-63

[23] Huang C.M., Meichle M., Salamon M.B. an Wayman C.M., Phil. Mag. A, 47 (1983) 9-30 and $31-62$

[24] Jordan L., Chandrasekaran M., Masse M. and Bouquet G., J. de Phys.,C2, 5 (1995) 489-493

[25] Otsuka K., Engineering Aspects of Shape Memory Alloys, Butterworth-Heinemann (1990) pp.3645

[26] Miyasaki S. and Wayman C.M., Acta Metall., 36 (1988) 181-192

[27] Miyasaki S., Kimura S. and Otsuka K., Phil. Mag. A, 57 (1988) 467-478

[28] Miyasaki S. and Otsuka K.,Phil. Mag. A, 50 (1984) 393-408

[29] Matsumoto M. and Honma T., New aspects of martensitic transformation (Sup. to Trans. JIM, vol. 17, 1976) pp.199

[30] Manosa Ll., Rios-Jara D., Ortin J., Planes A. and Bohigas X., J. Phys. : Cond. Matter, 4 (1992) $7059-66$

[31] Tamura H., Suzuki Y. and Todoroki T., Proc. ICOMAT 86, The Japan Institute of Metals (1987)

pp. 736-41

[32] Todoroki T., Engineering Aspects of Shape Memory Alloys, Butterworth-Heinemann (1990)

pp.315-29

[33] Treppmann D., Hornbogen E. and Wurzel D., J. de Phys.,C8 (1995) 569-74

[34] Miyasaki S., Nomura K. and A. Ishida, J. de Phys.,C8 (1995) 677-82

[35] Kawamura Y., Gyobu A., Horikawa H. and Saburi T.,J. de Phys.,C8 (1995) 683-88 